\title{
APPLICATION OF FRI MODEL TO SCC GROWTH ANALYSIS IN BWR COMPONENTS
}

\author{
Yoshihiko TANAKA \\ Tokyo Electric Power Company \\ e-mail: tanaka.yoshi@tepco.co.jp
}

Key Words: SCC, FRI model, $\mathrm{d} K / \mathrm{d} t, \mathrm{~d} K / \mathrm{d} a$

\begin{abstract}
Two methods of applying Fracture Research Institute Theoretical Stress Corrosion Cracking model (FRI model), the theoretical SCC model developed by Shoji and Suzuki [1], to SCC growth analysis in a cylindrical component with stress distribution are proposed.

FRI model has rarely been adapted to estimate SCC growth behavior in a real structural component in which the changing rate of stress intensity factor with time $(d K / d t)$ naturally changes as the crack grows. One of the reasons might be the difficulty of solving the equation consisting of many parameters including $d K / d t$.

In this paper, the authors propose two methods to conduct SCC growth analysis using FRI model. In Method 1 (Once-through method), the authors successfully applied FRI model to SCC growth analysis of 360 degree circumferential crack in a cylinder under the stress distribution by replacing $d K / d t$ with the changing rate of stress intensity factor with crack length $(d K / d a)$. Method 1 is so easy that it can be achieved only using Worksheet functions of EXCEL. Method 2 (Iteration method) is relatively difficult solving the complex equation at each crack growth step. The authors performed Method 2 using originally developed codes with VBA, Visual Basic for Applications, for EXCEL. Method 2 is applicable to two-dimensional crack (semi-elliptical crack) contrary to Method 1 that is only for a one-dimensional crack.

The details of Method 1 and Method 2, an example of application of FRI model to SCC growth analysis and the parameter study for FRI model are shown in this paper.
\end{abstract}

\author{
Shunichi SUZUKI \\ Tokyo Electric Power Company \\ e-mail: suzuki.shunichi@tepco.co.jp
}

\section{Conclusion}

1) The authors proposed the two calculation methods to estimate SCC growth behavior in a component having stress distribution based on FRI model.

2) The calculation results revealed the appreciable effect of $\mathrm{r}$ on the difference between $a$ with positive $\dot{K}$ and negative $K$ as well as magnitude of $\dot{a}$.

\section{Reference}

[1] Shoji T., Suzuki S., Ballinger R, Proc. 7th Int. Sym. on Environmental Degradation of Materials in Nuclear Power Systems - Water Reactors -, NACE, 1995, Houston, TX

[2] Shoji T. et al, , Proc. of ASME/JSME Pressure Vessel and Piping Conference (PVP-Vol.479), 2004, San Diego, California USA, ASME

[3] Suzuki S. ,Doctorate theses, Tohoku Univ. 2001 (in Japanese)

[4] Andresen P., Morra M.,Proc. 12th Int. Sym. on Environmental Degradation of Materials in Nuclear Power Systems - Water Reactors -, TMS, 2005, Snowbird, UT

[5] Eason D. et al., ibid.

[6] Tanaka Y., Suzuki S., Proc. of 18th Computational Mechanics Conference, 2005, Tsukuba, JSME (in Japanese)

[7] Homepage of Ministry of Economy, Trade and Industry

[8] "Fitness-for-Service, API Recommended Practice 579", First Edition, January 2002

[9] Japan Society of Mechanical Engineers, JSME S NA1-2004

[10] Suzuki S. et al., Proc. of ASME/JSME PVP Conference

Table 1 Approximation method for FRI model under a given stress distribution

\begin{tabular}{|c|c|c|}
\hline Parameters $\quad$ Model & Method 1 for FRI model: & Method 2 for FRI model:: \\
\hline Each calculation step is based on & $\begin{array}{c}\delta a \\
\text { Small crack length increment }\end{array}$ & $\begin{array}{c}\delta t \\
\text { Small time increment }\end{array}$ \\
\hline $\begin{array}{l}d K / d t \text { (or } K \text { ) for a small time } \\
\text { fraction, } t \text { through } t+\underline{\delta} t\end{array}$ & $\dot{K}=\frac{d K}{d t} \approx \frac{d K}{d a} \cdot \frac{d a}{d t}=\frac{d K}{d a} \cdot \dot{a}$ & $\dot{K}=\frac{K(a+\dot{a} \cdot \delta t)-K(a)}{\delta t}$ \\
\hline
\end{tabular}




\section{APPLICATION OF FRI MODEL TO SCC GROWTH ANALYSIS IN BWR COMPONENTS}

\author{
Yoshihiko TANAKA \\ Tokyo Electric Power Company \\ 4-1 Egasaki, Tsurumi, \\ Yokohama, Japan 230-8510 \\ Phone: +81-45-613-1111 \\ Fax: +81-45-613-7499 \\ e-mail: tanaka.yoshi@tepco.co.jp
}

\author{
Shunichi SUZUKI \\ Tokyo Electric Power Company \\ 4-1 Egasaki, Tsurumi, \\ Yokohama, Japan 230-8510 \\ Phone: +81-45-613-1111 \\ Fax: +81-45-613-7499 \\ e-mail: suzuki.shunichi@tepco.co.jp
}

Key Words: SCC, FRI model, $\mathrm{d} K / \mathrm{d} t, \mathrm{~d} K / \mathrm{d} a$

\begin{abstract}
Two methods of applying Fracture Research Institute Theoretical Stress Corrosion Cracking model (FRI model), the theoretical SCC model developed by Shoji and Suzuki [1], to SCC growth analysis in a cylindrical component with stress distribution are proposed.

FRI model has rarely been adapted to estimate SCC growth behavior in a real structural component in which the changing rate of stress intensity factor with time $(d K / d t)$ naturally changes as the crack grows. One of the reasons might be the difficulty of solving the equation consisting of many parameters including $d K / d t$.

In this paper, the authors propose two methods to conduct SCC growth analysis using FRI model. In Method 1 (Once-through method), the authors successfully applied FRI model to SCC growth analysis of 360 degree circumferential crack in a cylinder under the stress distribution by replacing $d K / d t$ with the changing rate of stress intensity factor with crack length $(d K / d a)$. Method 1 is so easy that it can be achieved only using Worksheet functions of EXCEL. Method 2 (Iteration method) is relatively difficult solving the complex equation at each crack growth step. The authors performed Method 2 using originally developed codes with VBA, Visual Basic for Applications, for EXCEL. Method 2 is applicable to two-dimensional crack (semi-elliptical crack) contrary to Method 1 that is only for a one-dimensional crack.

The details of Method 1 and Method 2, an example of application of FRI model to SCC growth analysis and the parameter study for FRI model are shown in this paper.
\end{abstract}

\section{Introduction}

Stress Corrosion Cracking (SCC) is one of the essential issues for appropriate life management of aged nuclear power plant components. Therefore, many codes and rules of Fitness-and-Service for Nuclear components in the world provide sufficient provisions for appropriate estimation of SCC. Most of them adopt the power law of the stress intensity factor $(K)$ simply assuming that SCC growth rate ( $d a / d t$ or $a$ ) should depend only on magnitude of $K$ : $d a / d t=C K^{n}$ where, $C$ and $n$ are constants. The power law, however, may not always give the appropriate prediction of SCC propagation behavior.

Shoji et al, have FRI model, presuming that $\mathrm{d} a / \mathrm{d} t$ should build upon the changing rate of $K(d K / d t$ or $\dot{K}$ ) as well as magnitude of $K$ [1]. FRI model consists of many parameters relating to the mechanical factor, the materials' corrosion resistance and the environment where the components work. In the past, some studies were conducted to predict the effect of the each parameter such as $d K / d t$ on SCC growth rate under the artificially fixed $d K / d t[2,3]$. The discrepancies between the power law based estimation for SCC growth rate and the actual data from the actual component might be reasonably explained by using FRI model. Some studies quantitatively estimated the key parameters of FRI model [4, 5]. However, FRI model has been rarely adapted to estimate SCC growth behavior in a real structural component in which $d K / d t$ naturally changes as the crack grows. One of the reasons might be the difficulty of solving the equation including the parameters, $a, K, d a / d t$ and $d K / d t$.

In this study, two methodologies for using FRI model for SCC growth calculation the components are proposed and value of the FRI model's important parameter $r$, the characteristic distance from crack tip, is assumed to be based on the SCC growth test under constant displacement. 


\section{Features of FRI model}

An example of the equations among various forms of FRI model for the plain strain condition is EQ (1).

$$
\begin{aligned}
\dot{a}= & \mathcal{K}_{a} \cdot\left(\frac{\beta \cdot \sigma_{y}}{E} \cdot \frac{n}{n-1}\right)^{m} \\
& \cdot\left(2 \cdot \frac{\dot{K}}{K}+\frac{\dot{a}}{r}\right)^{m} \cdot\left\{\ln \frac{\lambda\left(K / \sigma_{y}\right)^{2}}{r}\right\}^{\frac{m}{n-1}}
\end{aligned}
$$

where,

$a$ : Crack length

$\dot{a}$ : Crack growth rate $(=d a / d t)$

$K$ : Stress intensity factor

$\dot{K}$ : Change in $K$ with time $(=d K / d t)$

$\beta, \lambda$ : Dimensionless constants for plastic strain caluclation

$E$ : Young's modulus

$\sigma_{\mathrm{y}}:$ Yield stress

$n$ : Strain hardening exponent

$m$ :Slope of the oxidation rate decay curve in logarithmic scale

$\kappa_{a}$ : Crack tip oxidation rate constant

$r$ : Characteristic distance from crack tip

The third term of the right wing, $(2 K / K+\dot{a} / r)^{m}$, stands the followings.

1) $d a / d t$ (or $\dot{a}$ ) is controlled not only by $K$ but also by $\dot{K}$ and itself!

2) When $K$ decreases as crack grows under such stress distribution, da/dt decreases due not only to the reduction in magnitude of $K$ but also to the negative $\dot{K}(\dot{K}<0)$

3) Threshold of $K$ at which $\dot{a}$ becomes 0 ( $K_{\text {ISCC }}$ ) is as follows.

$$
K_{I S C C}=\sigma_{y} \cdot \sqrt{r / \lambda}
$$

4) Effect of $\dot{K}$ on da/d $t$ becomes more significant as $r$ gets larger.

The last item is especially interesting because it means that SCC growth rate with negative $\dot{K}$ might decrease even under fairly high $K$. This feature may bridge over the discrepancy between the estimation and the measured phenomena.

\section{Method 1: Once-through calculation method for SCC growth rate based on FRI model}

In EQ (1), $\dot{a}$ exists in both of the wings. That makes EQ (1) difficult to be directly solved. The authors approximate $\dot{K}$ as follows [6].

$$
\dot{K}=\frac{d K}{d t} \approx \frac{d K}{d a} \cdot \frac{d a}{d t}=\frac{d K}{d a} \cdot \dot{a}
$$

EQ (3) is substituted for $K$ in EQ (1) to make EQ (4). " $a$ ” exists in the both wings.

$$
\begin{aligned}
\dot{a} \approx & \boldsymbol{K}_{a} \cdot\left(\frac{\beta \cdot \sigma_{y}}{E} \cdot \frac{n}{n-1}\right)^{m} \cdot \dot{a}^{m} \\
& \cdot\left(\frac{2}{K} \cdot \frac{d K}{d a}+\frac{1}{r}\right)^{m} \cdot\left\{\ln \frac{\lambda\left(K / \sigma_{y}\right)^{2}}{r}\right\}^{\frac{m}{n-1}}
\end{aligned}
$$

Well organization of EQ (4) leads to EQ (5).

$$
\begin{aligned}
\dot{a} & \approx \boldsymbol{K}_{a}{ }^{\frac{1}{1-m}} \cdot\left(\frac{\beta \cdot \sigma_{y}}{E} \cdot \frac{n}{n-1}\right)^{\frac{m}{1-m}} \\
& \cdot\left(\frac{2}{K} \cdot \frac{d K}{d a}+\frac{1}{r}\right)^{\frac{m}{1-m}} \cdot\left\{\ln \frac{\lambda\left(K / \sigma_{y}\right)^{2}}{r}\right\}^{\frac{m}{(n-1) \cdot(1-m)}}
\end{aligned}
$$

Most of the codes and rules provide $K$ and resultantly $d K / d a$ as simple functions of $a$. It is, therefore, easy to obtain SCC growth rate based on FRI model by using EQ (5).

\section{Method 2: Iterative method for SCC growth rate based on FRI model}

For EQ (1), it is difficult to achieve simultaneous convergence of $K, d a / d t$ and $d K / d t$. Therefore, the authors assumed the points below.

1) $K$ is supposed to be constant in the small time fraction, from $t$ to $t+\delta t$.

2) Since $K$ is function of $a, K$ is as follows in the small time fraction.

$$
\dot{K}=\frac{K(a+\dot{a} \cdot \delta t)-K(a)}{\delta t}
$$

These assumptions enable solving EQ (1) by iteration to find SCC growth rate $(a)$ in each time step, e.g. from $t$ to $t+\delta t$. Substitution of EQ (6) for $K$ in EQ (1) leads to EQ (7). 


$$
\begin{gathered}
\dot{a}=K_{a} \cdot\left(\frac{\beta \cdot \sigma_{y}}{E} \cdot \frac{n}{n-1}\right)^{m} \\
\cdot\left(2 \cdot \frac{K(a+\dot{a} \cdot \delta t)-K(a)}{K \cdot \delta t}+\frac{\dot{a}}{r}\right)^{m} \\
\cdot\left\{\ln \frac{\lambda\left(K / \sigma_{y}\right)^{2}}{r}\right\}^{\frac{m}{n-1}}
\end{gathered}
$$

Note: $\quad K=K(a)$

Features of Method 1 and 2 are shown in Table 1.

\section{Merit and Demerit of Method 1 and 2}

Since Method 2 conducts iterative calculation, it tends to take a much longer calculation time than Method 1. However, for Method 1, it is necessary to determine value of $d K / d a$ for the all calculation steps before starting the calculation. Namely, $\delta a$, the crack length increments for each step, should be previously decided and (in most case) kept constant. This feature of Method 1 is not a demerit for 1-dimensional crack such as 360 degree circumferential crack on cylinder surface. However, for semi-elliptical crack (2-dimensional crack), Method 2 works well because the same $\delta$, the time increments for each step, can be applied both to the crack depth and crack length.

\section{An example of SCC growth rate calculation in BWR core shroud by Method 2}

For calculation, values for the many parameters in EQ (1) need to be set. Shoji et al applied the values in "Condition 1" shown in Table 2 for the parameters in FRI model [2]. The characteristic distance from crack tip, $r$, is assumed to be $10^{-5} \mathrm{~m}$, several ten $\%$ of diameter of the grains. On the other, S. Suzuki adopted the greater $r$, order of $10^{-4} \mathrm{~m}$ as shown in "Condition 2 " in the same table [3]. Accordingly, "Condition 1A" and "Condition 1B", of which $r$ are 10 times and 100 times larger than the standard respectively, were added to find the effect of $r$.

In this study, these conditions are used for crack growth analysis. The assumed component for the analysis is the H6a weld in the reactor core shroud made of stainless steel of which shape is cylinder. Shape of the initial crack is $360^{\circ}$-circumferential crack of $300 \mu \mathrm{m}$ depth on the external surface of the shroud (Fig. 1). The stress distribution due to the weld along the thickness is shown in Fig. 2 [7]. $K$ was given using the API code for circumferential crack [8]. Then $\dot{K}$ and da/d $t$ were iteratively calculated using EQ (1) and (6) with $K$ in each sufficiently small time fraction $\delta t$ in which $K$ can be assumed to be constant.
Trends of $\dot{K}, K$ and $\dot{a}$ of Condition 2 are in Fig. 3 . The $K$ vs. $d a / d t$ curves are shown in Fig. 4 . The curve from JSME code, S NA1-2004 [9], is also plotted for reference. It exactly shows the significance of FRI model: da/dt differ under the same $K$ because of different $\dot{K}$. In the early stage, $K$ increases as the crack advances, keeping $\dot{K}>0$. Later, $K$ passes the peak and begins to decrease producing $\dot{K}<0$. The locus of $\dot{K}<0$ locates under that of $\dot{K}>0$. Condition 2 with greater $r$ gives more obvious difference between the two loci. This phenomenon is reasonably explained by the third term of right-hand of EQ (1): the greater $r$, the greater effect of $\dot{K}$ on da/dt. Fig. 5 shows the trends of crack depth, $a$ vs. time. The results from FRI model with the different parameters of Condition 1 and 2 in Table 2 are clearly different. It is suggested that the difference in the imaginary numbers, $\lambda$ and $r$, produce such different results. As is shown in the figure, the result from FRI model of Condition 2 almost coincides with the crack depth observed in the actual plant [7].

\section{Estimation of the characteristic distance from crack tip $r$ based on WOL test result}

As discussed in the previous section, the characteristic distance from crack tip $r$ affects the difference between the loci of $\dot{K}>0$ and $\dot{K}<0$.

FRI model of Condition 1, 1A and 1B have different $r$ respectively as shown in Table 2. SCC growth calculations were performed with these three conditions to figure out the effect of $r$. The $d a / d t$ - $K$ curves obtained from the calculation results are shown in Fig. 6. It is obvious that $r$ has significant effect on the difference between the loci for positive $\dot{K}$ and negative $\dot{K}$ as well as magnitude of $a$.

Among experimental SCC growth data, the authors found that some of SCC growth rates data with WOL specimen, loaded by displacement controlled stress, rapidly fell down to less $6 \mathrm{x}$ $10^{-13} \mathrm{~m} / \mathrm{s}, 0.2 \mathrm{~mm} /$ year, or less at fairly high $\mathrm{Ks}$ of around $33 \mathrm{MPam}^{0.5}[10]$. Table 3 shows the results including $K$ and $d K / d a \mathrm{MPam}^{0.5} / \mathrm{m}$. Suppose that 0.2 $\mathrm{mm} / \mathrm{y}$ is small enough, $r$ is approximated as follows. 


$$
\begin{aligned}
\dot{a} \approx & K_{a} \frac{1}{1-m} \cdot\left(\frac{\beta \cdot \sigma_{y}}{E} \cdot \frac{n}{n-1}\right)^{\frac{m}{1-m}} \\
& \cdot\left(\frac{2}{K} \cdot \frac{d K}{d a}+\frac{1}{r}\right)^{\frac{m}{1-m}} \cdot\left\{\ln \frac{\lambda\left(K / \sigma_{y}\right)^{2}}{r}\right\}^{\frac{m}{(n-1) \cdot(1-m)}} \\
= & 0.2 \mathrm{~mm} / \mathrm{y} \approx 0 \mathrm{~mm} / \mathrm{y}
\end{aligned}
$$

In the above equation, only the third term on the right hand side can be 0 . Therefore,

when $\dot{a} \rightarrow 0$, then

$$
r \rightarrow-\left(\frac{2}{K} \cdot \frac{d K}{d a}\right)^{-1}
$$

Using EQ (9), and TEST_1 and TEST_2 data in Table 3 give $r$ of around $16 \mathrm{~mm}$ (Table 2). Those are incredibly greater than several ten percent of the grain size assumed in Condition 1 and 2. The main reasons for the difference should be that $a$ is not strictly zero. Also displacement controlled type stress in WOL specimen might produce remarkable difference provided that $r$ of Condition 1 and 2 were determined based on test result mainly with CT specimen that is usually given by Load controlled type stress.

Anyway, the actual plant components are assumed to have Elastic follow-up type stress, an intermediate between the former two type stresses. Therefore, the possibility of the actual components with higher $r$ than those supposed in Condition 1 and 2 can not be ruled out completely, though it is difficult to establish quantitative information.

\section{Conclusion}

1) The authors proposed the two calculation methods, EQ (5) and (7) to estimate SCC growth behavior in a component having stress distribution based on FRI model.

2) The calculation results revealed the appreciable effect of $\mathrm{r}$ on the difference between $a$ with positive $\dot{K}$ and negative $\dot{K}$ as well as magnitude of $\dot{a}$.

\section{Reference}

[1] Shoji T., Suzuki S., Ballinger R, "Theoretical Prediction of SCC Growth Behavior," Proc. 7th Int. Sym. on Environmental Degradation of Materials in Nuclear Power Systems - Water Reactors -, NACE, 1995, Houston, TX

[2] Shoji T. et al, "Modeling and Quantitative
Prediction of Environmentally Assisted

Cracking Based Upon a Deformation-Oxidation Mechanism", Proc. of ASME/JSME Pressure Vessel and Piping Conference (PVP-Vol.479), 2004, San Diego, California USA, ASME

[3] Suzuki S. , Doctorate theses, Tohoku Univ. 2001 (in Japanese)

[4] Andresen P., Morra M., "Effects of Positive and Negative $\mathrm{d} K / \mathrm{d} a$ on SCC Growth rates," Proc. 12th Int. Sym. on Environmental Degradation of Materials in Nuclear Power Systems - Water Reactors -, TMS, 2005, Snowbird, UT

[5] Eason D. et al., "Evaluation of the Fracture Research Institute Theoretical Stress Corrosion Cracking Model,” ibid.

[6] Tanaka Y., Suzuki S., "Study on Applicability of Theoretical EAC Model to SCC Growth Analysis for BWR environment", Proc. of 18th Computational Mechanics Conference, 2005, Tsukuba, JSME (in Japanese)

[7] Homepage of Ministry of Economy, Trade and Industry, http://www.meti.go.jp /kohosys/committee/summary /0001368/0001.html

[8]American Petroleum Institute, "Fitness-for-Service, API Recommended Practice 579", First Edition, January 2002

[9] Japan Society of Mechanical Engineers, Code for Nuclear Power Generation Facilities, JSME S NA1-2004

[10] Suzuki S. et al., "Study on the SCC Growth of Alloy 182 under Constant Displacement Condition in High Temperature Oxygenated Water", Proc. of ASME/JSME PVP Conference (PVP-Vol.479), 2004, San Diego, California USA, ASME 
Table 1 Approximation method for FRI model under a given stress distribution

\begin{tabular}{|c|c|c|c|}
\hline Parameters & $\begin{array}{l}\text { Method } 1 \text { for FRI model: } \\
\text { “ Once-through” }\end{array}$ & $\begin{array}{l}\text { Method } 2 \text { for FRI model:: } \\
\text { “ Iterative” }\end{array}$ & $\begin{array}{c}\text { Conservative } \\
\text { power of } \boldsymbol{K} \text { model } \\
\text { for comparison } \\
\end{array}$ \\
\hline $\begin{array}{l}\text { Each calculation } \\
\text { step is based on }\end{array}$ & $\begin{array}{c}\delta a \\
\text { Small crack length increment }\end{array}$ & $\begin{array}{c}\delta t \\
\text { Small time increment }\end{array}$ & Anyway \\
\hline $\begin{array}{l}\text { K for a small } \\
\text { crack length } \\
\text { fraction, } a \\
\text { through } a+\underline{\delta} a\end{array}$ & $\begin{array}{l}\dot{a}=\text { Constant } \\
K=\text { Constant }\end{array}$ & --- & $K=$ Constant \\
\hline $\begin{array}{l}K \text { for a small } \\
\text { time fraction, } t \\
\text { through } t+\underline{\delta} t\end{array}$ & --- & $\begin{array}{l}\dot{a}=\text { Constant } \\
K=\text { Constant }\end{array}$ & $K=$ Constant \\
\hline $\begin{array}{l}d K / d t \text { (or } K \text { ) } \\
\text { for a small time } \\
\text { fraction, } t \\
\text { through } t+\underline{\delta} t\end{array}$ & $\begin{array}{l}\dot{K}=\frac{d K}{d t} \approx \frac{d K}{d a} \cdot \frac{d a}{d t}=\frac{d K}{d a} \cdot \dot{a} \\
* \text { Since } K \text { is a function of } a, \mathrm{~d} K / \mathrm{d} a \\
\text { is easily obtained }\end{array}$ & $\dot{K}=\frac{K(a+\dot{a} \cdot \delta t)-K(a)}{\delta t}$ & $\begin{array}{l}\text { The model can not } \\
\text { consider the effect } \\
\text { of } d K / d t\end{array}$ \\
\hline $\begin{array}{l}d a / d t \quad(\text { or } a) \\
\text { for a small time } \\
\text { fraction, } t \\
\text { through } t+\underline{\delta} t\end{array}$ & $\begin{aligned} \dot{a} \approx & \boldsymbol{K}_{a} \frac{1}{1-m} \cdot\left(\frac{\beta \cdot \sigma_{y}}{E} \cdot \frac{n}{n-1}\right)^{\frac{m}{1-m}} \\
& \cdot\left(\frac{2}{K} \cdot \frac{d K}{d a}+\frac{1}{r}\right)^{\frac{m}{1-m}} \\
& \cdot\left\{\ln \frac{\lambda\left(K / \sigma_{y}\right)^{2}}{r}\right\}^{\frac{m}{(n-1) \cdot(1-m)}}\end{aligned}$ & $\begin{array}{r}\dot{a}=K_{a} \cdot\left(\frac{\beta \cdot \sigma_{y}}{E} \cdot \frac{n}{n-1}\right)^{m} \\
\cdot\left(2 \cdot \frac{K(a+\dot{a} \cdot \delta t)-K(a)}{K \cdot \delta t}+\frac{\dot{a}}{r}\right)^{m} \\
\cdot\left\{\begin{array}{l}\lambda\left(K / \sigma_{y}\right)^{2} \\
r\end{array}\right\}^{\frac{m}{n-1}}(7) \\
\text { Note: } K=K(a)\end{array}$ & $\dot{a}=A \cdot K^{n}$ \\
\hline Calculation & $\begin{array}{l}\text { Solve EQ (5) in each step, e.g. } \\
\text { through } a \text { through } a+\delta a \text { is easy } \\
\text { 1) } a \text { : crack length at the end of the } \\
\text { previous step } \\
\text { 2) } \delta a \text { : sufficiently small crack } \\
\text { length increment for } a \text { of the } \\
\text { previous step } \\
\text { 3) Range of the current step is } \\
\text { between } a \text { through } a+\delta a \\
\text { 4) } K \text { for the current step is } \\
\text { determined from crack length } a \\
\text { of the previous step and the } \\
\text { stress distribution } \\
\text { 5) Iteration is not needed }\end{array}$ & $\begin{array}{l}\text { Solve EQ(7) by iterative calculation } \\
\text { in each step, e.g. } t \text { through } t+\delta t \\
\text { 1) } t \text { : time point at the end of the } \\
\text { previous step } \\
\text { 2) } \delta t \text { : sufficiently small time } \\
\text { increment for } a \text { of the previous } \\
\text { step } \\
\text { 3) Range of the current step is } \\
\text { between } t \text { through } t+\delta t \\
\text { 4) } K \text { for the current step is } \\
\text { determined from crack length } a \\
\text { of the previous step and the } \\
\text { stress distribution } \\
\text { 5) Initial values of } \dot{a} \text { and } \dot{K} \text { for } \\
\text { iteration are those of the } \\
\text { previous step }\end{array}$ & Easy \\
\hline $\begin{array}{l}\text { Applicability for } \\
\text { 360- } \\
\text { degree-circumfe } \\
\text { rential crack }\end{array}$ & Easy & Possible & Practically used \\
\hline $\begin{array}{l}\text { Applicability for } \\
\text { semi-elliptical } \\
\text { crack }\end{array}$ & Impossible? & Possible & Practically used \\
\hline
\end{tabular}


Table 2 FRI model's parameters for crack growth analysis

\begin{tabular}{|c|c|c|c|c|}
\hline Parameters & Condition $1^{[2]}$ & Condition 1A & Condition 1B & Condition 2 $^{[3]}$ \\
\hline$\beta$ & 5.08 & Same as left & Same as left & 5.08 \\
\hline$\lambda$ & 0.30 & Same as left & Same as left & $0.050^{*}$ \\
\hline$n$ & 1.70 & Same as left & Same as left & 4.1 \\
\hline$\kappa_{a}, \mathrm{~m} / \mathrm{s}^{1+m}$ & $7.798 \mathrm{E}-7$ & Same as left & Same as left & $8.720 \mathrm{E}-07$ \\
\hline$m$ & 0.633 & Same as left & Same as left & 0.5 \\
\hline$r, \mathrm{~m}$ & $1.0 \mathrm{E}-5$ & $1.0 \mathrm{E}-4$ & $1.0 \mathrm{E}-3$ & $1.7 \mathrm{E}-4$ \\
\hline $\mathrm{E}, \mathrm{MPa}$ & 190000 & Same as left & Same as left & 173074 \\
\hline$\sigma_{y}, \mathrm{MPa}$ & 180 & Same as left & Same as left & 180 \\
\hline
\end{tabular}

* The original figure, 0.025, was doubled for a sight in Fig. 3

Table 3 WOL test result and assumed " $r$ " [10]

\begin{tabular}{|c|c|c|c|c|}
\hline & Test_1 & Test_2 & Test_3 & Test_4 \\
\hline $\mathrm{d} a / \mathrm{d} t, \mathrm{~m} / \mathrm{s}$ & $4.0 \mathrm{E}-12$ & $4.0 \mathrm{E}-12$ & $3.9 \mathrm{E}-12$ & $6.0 \mathrm{E}-12$ \\
\hline $\mathrm{K}, \mathrm{MPam}^{0.5}$ & 33.3 & 33.2 & 14.9 & -488.0 \\
\hline $\mathrm{d} K / \mathrm{d} a, \mathrm{MPam}{ }^{0.5} / \mathrm{m}$ & -1033.7 & -1033.2 & -438.6 & $1.66 \mathrm{E}-2$ \\
\hline$r^{\prime \prime}$ obtained by EQ (9) & & & $1.70 \mathrm{E}-2$ & \\
\hline
\end{tabular}




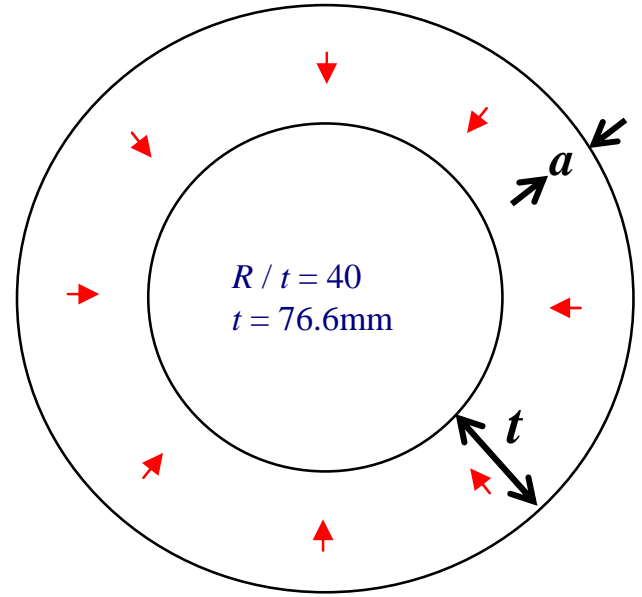

Fig. 1 Assumed structure and crack: Circumferential crack grows from external surface

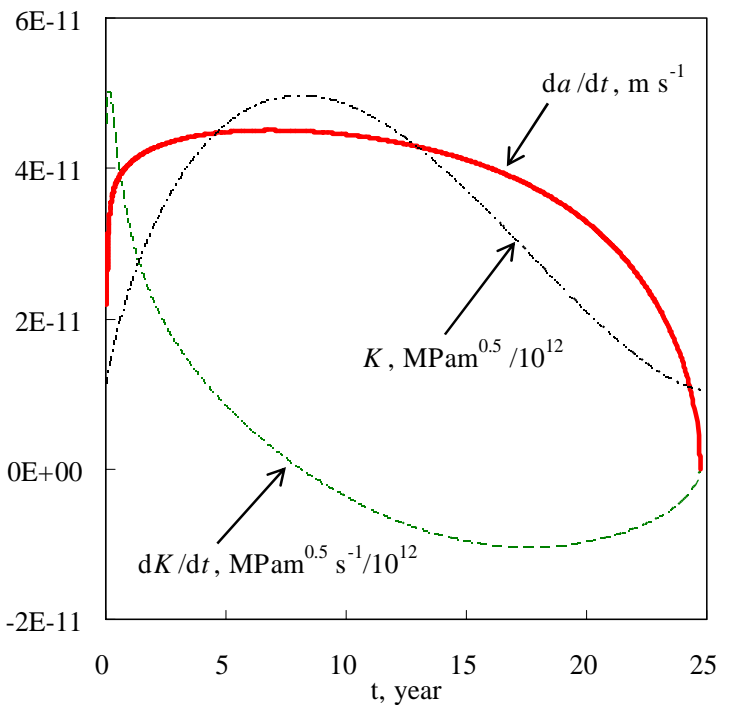

Fig. 3 Trends of $K, \mathrm{~d} a / \mathrm{d} t$ and $\mathrm{d} K / \mathrm{d} t$ FRI model of Condition 2

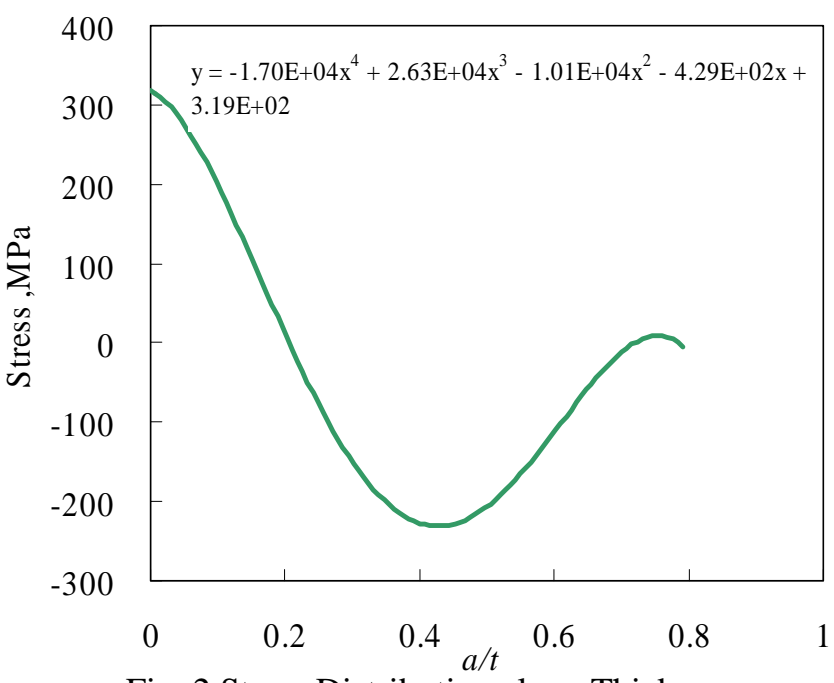

Fig. 2 Stress Distribution along Thickness

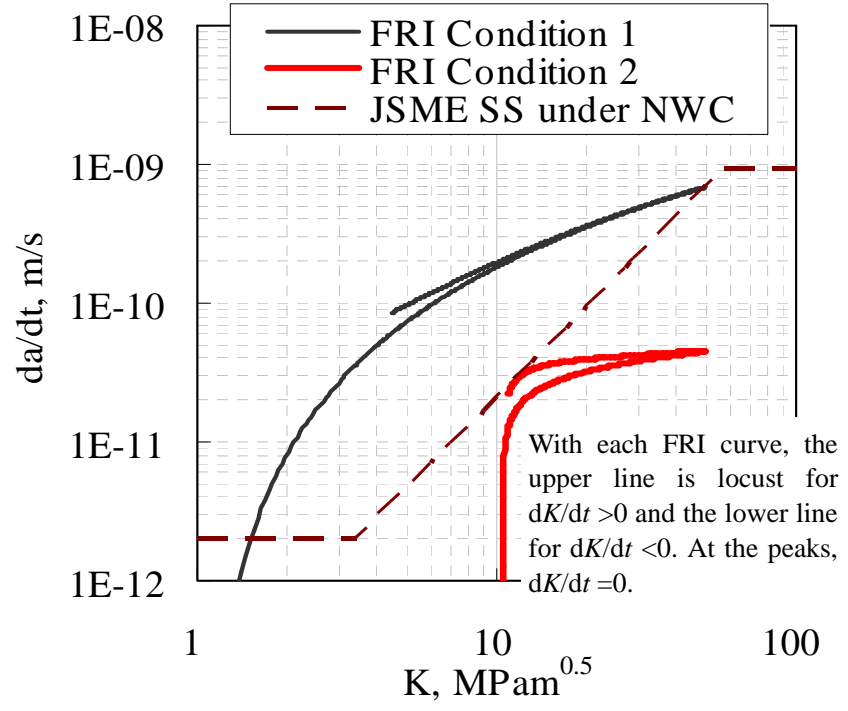

Fig. $4 \mathrm{~K}$ vs. d $a / \mathrm{d} t$ diagram of FRI model and JSME diagram 


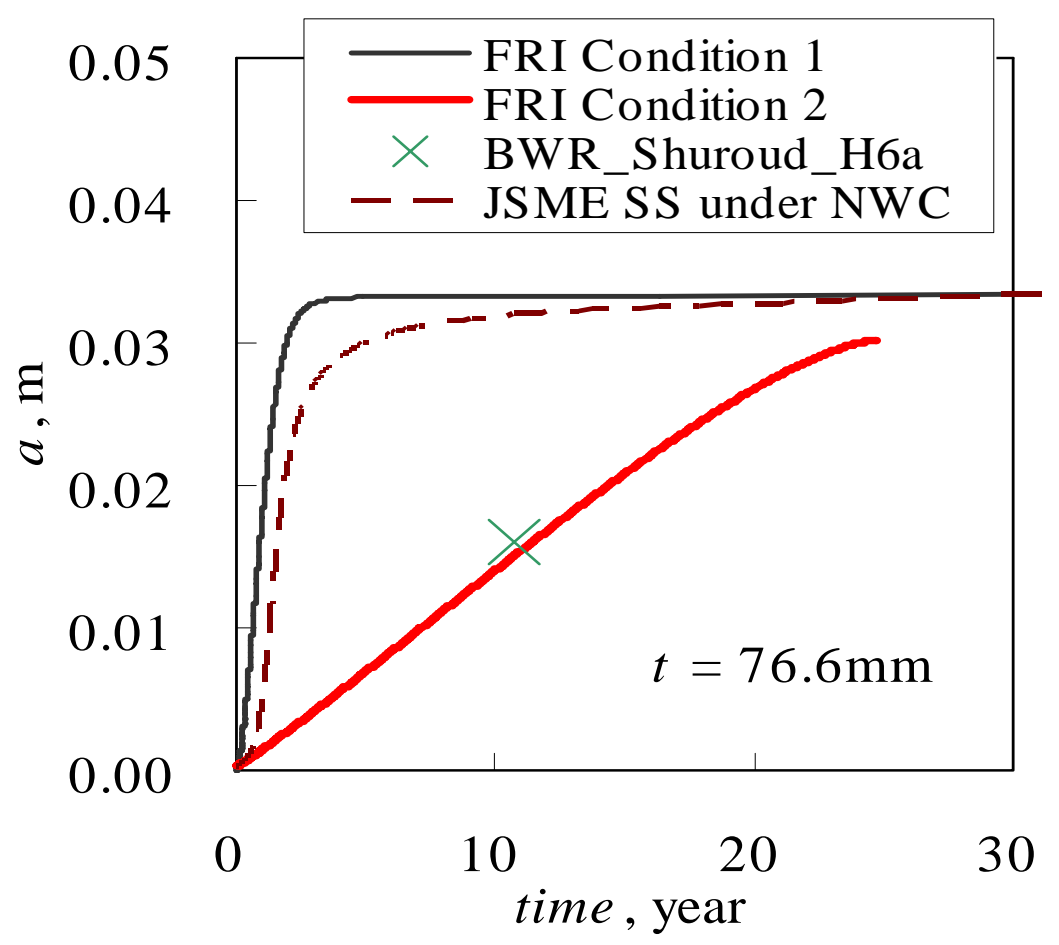

Fig. 5 Trends of crack length by FRI model

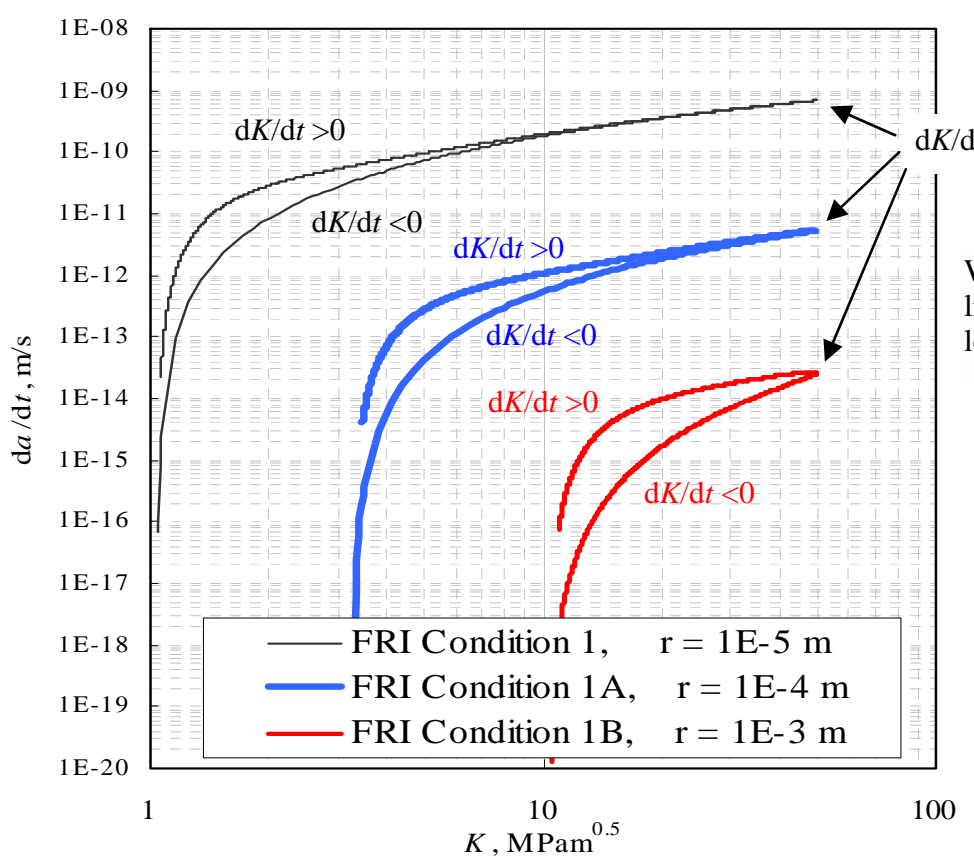

With each FRI curve, the upper line is locust for $\mathrm{d} K / \mathrm{d} t>0$ and the lower line for $\mathrm{d} K / \mathrm{d} t<0$.

Fig. 6 Effect of $r$ on $K$ vs. da/d $t$ diagram 